Contributions to Game Theory and Management, XIV, 49-58

\title{
On the Value of the Preexisting Knowledge in an Optimal Control of Pollution Emissions*
}

\author{
Angelina Chebotareva, Su Shimai, Sophia Tretyakova \\ and Ekaterina Gromova \\ St. Petersburg State University, \\ 7/9 Universitetskaya nab., Saint Petersburg, 199034, Russia \\ E-mail: angel.xe99@gmail.com, brucesusm@gmail.com, \\ docsophy@gmail .com, e.v.gromova@spbu.ru
}

\begin{abstract}
For a classical differential game of pollution control, we consider how the possession of specific information would impact the payoff of some players compared to cases in which the knowledge of information is incomplete. To measure the resulting discrepancy, we use the notion of value of information (VoI). Specifically, we study two scenarios, one in which the role of knowledge about the terminal cost is studied, and the other one, in which we analyze the influence of knowledge about the exact value of the upper bound on control. For each case, we obtain explicit analytical expressions for the payoff functions. These functions are used to quantify the exact value of information.
\end{abstract}

Keywords: differential game, pollution problems, value of information, optimal control and trajectory.

\section{Introduction}

In the paper two new models of the pollution emissions control is considered and the new characteristic such that the value of information is suggested.

First of all, we have to admit that the announcement of policy to the business does have the character of pre-informing which means that the government usually won't take an action abruptly in order to keep a healthy and stable development of the business. However, we should not rule out the possibility of the occurrence of such scenario. Especially, under the current harsh competitive environment, the company or person would always go for the maximal profit without seriously considering side effects they may cause to the society or nature. For example, for the paper mill, since the amount of orders directly works on their profit, it's quite difficult for them to put the wastewater treatment on the first priority and meanwhile, they are still taking the original strategy to emit enormous pollution, which also shows lots of production is underway. In this case, the appropriate interference of penalty policy at the end of a period without previously informing could give them a strong alarm and largely guarantee the normal operation of production. The interesting thing is if the company somehow is aware of such penalty at the beginning of the production period, so instead of emitting unlimited pollution, they are making effort to reduce it and keeping the good function of business at the same time. Then what is the difference of their total payoff during a fixed time?

Besides, the authority could flexibly adjust the threshold of emission rate according to the real situation to amplify the effectiveness. The obvious case is that the emission control alters a lot due to the change of season. And what if the company learns

${ }^{*}$ The reported study was funded by RFBR and DFG, project number 21-51-12007.

https://doi.org/10.21638/11701/spbu31.2021.04 
the change of upper bound of emission rate beforehand, how will the company react in contract to the unknown case? This paper is specially dealing with these two cases in terms of the separate modelling.

\section{Value of information for uncertainty of terminal cost}

The model of control over the volumes of pollutant emission is being considered (Dockner et al., 2000). The total payoff is obtained with a terminal cost corresponding to a fine for pollution at the last moment of the product period. Under this circumstance, we study the value of information, which shows how the awareness of terminal cost would affect the payoff of the business owner.

As (Tur and Gromova, 2018) shows one cooperative solution in a differential emission control game, (Gromov and Gromova, 2017) describes the conditions for decision of optimal control and (Gromova, 2016) defines the cooperative solution in differential games of three players, we formulate our model and computation as following.

Suppose there are 3 players in our case. It is assumed that the production volumes are directly proportional to the volume of environmental pollution. The strategy of player $i$ can be viewed as the decision of the pollution rate per unit time, $u_{i} \in\left[0, b_{i}\right]$. The dynamic is defined by the equation:

$$
\dot{x}(t)=\sum_{i=1}^{3} u_{i}(t), \quad x\left(t_{0}\right)=x_{0}, \quad t \in\left[t_{0}, T\right] .
$$

Suppose the penalty at the terminal moment is also straight proportional to the level of pollution $X(T)$. Then the payoff of player $i$ is a mixed Bolza function:

$$
K_{i}\left(x_{0}, T-t_{0}, u\right)=\int_{t_{0}}^{T}\left(\left(b_{i}-\frac{1}{2} u_{i}(t)\right) u_{i}(t)-d_{i} x\right) d t-D_{i} x(T),
$$

where $u=\left\{u_{1}, u_{2}, u_{3}\right\}, b_{i} \geq 0$ is the ratio of total benefit of player $i$ from the production; $d_{i} \geq 0$ is the expense of elimination of a unit of total pollution for player $i ; D_{i} \geq 0$ is the amount of the penalty per unit of pollution. We will assume that $t_{0}=0$ in the following calculation. And the players initially agree on the use of optimal controls which will maximize the total gain,

$$
\sum_{i=1}^{3} K_{i}\left(x_{0}, T, u\right) \rightarrow \max _{u}
$$

For simplicity, let us define several abbreviations,

$$
\begin{aligned}
& b_{123}=b_{1}+b_{2}+b_{3}, \quad d_{123}=d_{1}+d_{2}+d_{3} \\
& D_{123}=D_{1}+D_{2}+D_{3}, \quad D_{i j}=D_{i}+D_{j} .
\end{aligned}
$$

The maximization problem can be solved according to (Gamkrelidez, 1999), (Pontryagin, 1987).

The Hamiltonian function is:

$$
H(x, u, \psi)=\sum_{i=1}^{3}\left[\left(\left(b_{i}-\frac{1}{2} u_{i}\right) u_{i}\right)-D_{i} x(T)\right]+\psi\left(u_{1}+u_{2}+u_{3}\right) .
$$


The optimal control has to maximize the Hamiltonian function by complying with necessary condition:

$$
\begin{gathered}
\frac{\partial H}{\partial u_{i}}=b_{i}-u_{i}+\psi=0, \\
u_{i}^{*}=b_{i}+\psi
\end{gathered}
$$

Since the second derivative of $H$ at $u=u_{i}^{*}$ is negative, we confirm that the optimal control $u_{i}^{*}$ does maximize the Hamiltonian function,

$$
\frac{\partial^{2} H}{\partial u_{i}^{2}}=-1<0
$$

The adjoint equations:

$$
\frac{\partial \psi}{\partial t}=-\frac{\partial H}{\partial x}=d_{123} .
$$

Hence,

$$
\begin{gathered}
\psi(t)=d_{123} t+C \\
\psi(T)=\left.\frac{\partial H}{\partial x(t)}\right|_{t=T}=-D_{123}=d_{123} T+C, \\
\psi(t)=(t-T) d_{123}-D_{123} .
\end{gathered}
$$

The optimal control is:

$$
u_{i}^{*}=b_{i}-(T-t) d_{123}-D_{123}, \quad i=1,2,3 .
$$

The optimal trajectory is:

$$
x^{*}(t)=\left(b_{123}-D_{123}\right) t-d_{123} T t+\frac{1}{2} t^{2} d_{123}+x_{0} .
$$

Let us introduce additional constraints on the parameters of the model, which guarantee that the result for some other auxiliary control optimization problems are admissible and belongs to the compact set $\left[0, b_{i}\right]$ :

$$
\begin{gathered}
D_{i} \in\left[0, \min \left(b_{1}-D_{123}, b_{2}-D_{123}, b_{3}-D_{123}\right)\right] \\
d_{i} \in\left[0, \frac{\min \left(b_{1}, b_{2}, b_{3}\right)-D_{123}}{T}-d_{123}\right], \quad i=1,2,3 .
\end{gathered}
$$

Since the calculation of optimal control and optimal trajectory for each player shares the same character, for the following two cases, we are trying to generalize the problem and only concentrate on the performance of one player, s.t. player 1 .

\subsection{Terminal cost is known}

It is well-known (Basar and Olsder, 1998) that for linear-quadratic differential game (1), (2) Nash equilibrium exists and is unique. Now we can similarly calculate the Nash equilibrium using the Pontryagin maximum principle (Pontryagin, 1987). Then the Nash equilibrium have the form:

$$
u_{i}^{N E}(t)=b_{i}-(T-t) d_{i}-D_{i}, \quad i=1,2,3 .
$$


Now we calculate the value of the payoff of the business owner in the Nash equilibrium, substituting the obtained control into formula (2):

$$
\begin{aligned}
K_{1}^{N E} & =K_{1}\left(x_{0}, T, u^{N E}\right) \\
& =-x_{0}\left(T d_{1}+D_{1}\right)+D_{1} T\left(D_{123}-b_{123}+T d_{123}\right)+\frac{T\left(b_{1}^{2}-D_{1}^{2}\right)}{2} \\
& +\frac{T^{2}\left(d_{1} D_{23}-d_{1} b_{123}-D_{1} d_{123}\right)}{2}+\frac{T^{3}\left(d_{1}^{2}+2 d_{1} d_{2}+2 d_{1} d_{3}\right)}{6} .
\end{aligned}
$$

For the rest of the players $i=2,3$, the value of the payoffs in the Nash equilibrium can be acquired by cyclic permutation of the indices in (1).

\subsection{Terminal cost is unknown}

Suppose the player does not know that at the last moment of the game he will be assigned a terminal penalty $D_{i} x(T)$. We suppose $D_{i}=0$, although in fact it's not. Then the payoff function for the player,

$$
\bar{K}_{1}\left(x_{0}, T, u\right)=\int_{t_{0}}^{T}\left(\left(b_{1}-\frac{1}{2} u_{1}(t)\right) u_{1}(t)-d_{1} x\right) d t .
$$

Correspondingly, the player will use the controls below as to Nash equilibrium:

$$
u_{1}^{* N E}(t)=b_{1}-(T-t) d_{1} .
$$

And the corresponding trajectory follows:

$$
x^{* N E}(t)=b_{123} t-d_{123} T t+\frac{1}{2} t^{2} d_{123}+x_{0} .
$$

While the player is not aware of terminal cost, it actually exists. Thus the player is still obligatory to pay penalty and the calculation of player's payoff has to follow (2) as well:

$$
\begin{aligned}
K_{1}^{* N E} & =-D_{1} x_{0}-T\left(D_{1} b_{123}+d_{1} x_{0}-\frac{b_{1}^{2}}{2}\right) \\
& +\frac{T^{2}\left(D_{1} d_{123}-d_{1} b_{123}\right)}{2}+\frac{T^{3} d_{1}\left(d_{123}+d_{23}\right)}{2} .
\end{aligned}
$$

Before calculating value of information, we would like to compare the control and trajectory corresponding to the Nash equilibrium for the previous cases. We assume that $b_{1}=300, b_{2}=305, b_{3}=303, T=20, d_{1}=2, d_{2}=3, d_{3}=4, D_{1}=3, D_{2}=$ $6, D_{3}=5, x_{0}=5$ (Note that these parameters have to satisfy the conditions above). With current setting, we notice that there is no significant variance between them. As illustrated below, the curve of controls and trajectory in first case are quite adjacent to another. We will quantify such difference later.

\subsection{Evaluation of value of information - VI}

In order to evaluate the characteristic value of information (VI) first suggested by (Raiffa and Schlaifer, 1961) for problem with incomplete information, we propose 


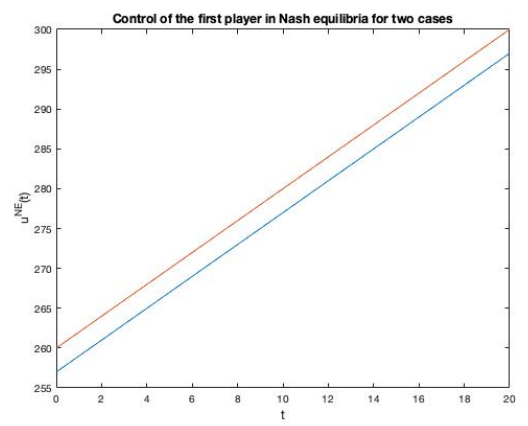

Fig. 1. Control of the first player in Nash equilibria for two cases

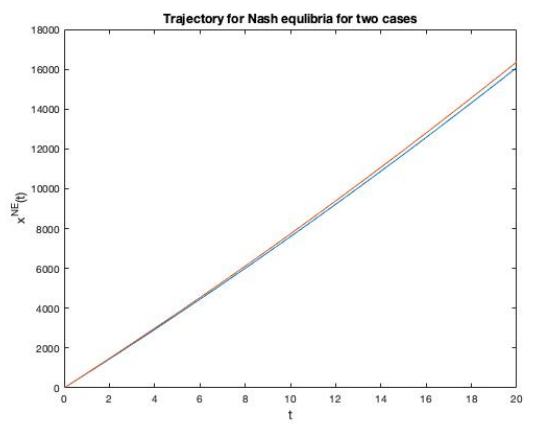

Fig. 2. Trajectory for Nash equilibria for two cases

the following characteristic $\frac{1}{N V I}$,

$$
\begin{aligned}
\frac{1}{N V I_{1}} & =\frac{K_{1}^{N E}}{K_{1}^{N E}-K_{1}^{* N E}} \\
& =\frac{-2 x_{0}\left(T d_{1}+D_{1}\right)}{T\left(D_{1}\left(D_{123}+D_{23}\right)+T d_{1} D_{23}\right)}+\frac{2 D_{1}\left(D_{123}-b_{123}\right)}{D_{1}\left(D_{123}+D_{23}\right)+T d_{1} D_{23}} \\
& +\frac{b_{1}^{2}-D_{1}^{2}}{D_{1}\left(D_{123}+D_{23}\right)+T d_{1} D_{23}}+\frac{T\left(d_{1} D_{23}-d_{1} b_{123}+D_{1} d_{123}\right)}{D_{1}\left(D_{123}+D_{23}\right)+T d_{1} D_{23}} \\
& +\frac{T^{2}\left(d_{1}^{2}+2 d_{1} d_{2}+2 d_{1} d_{3}\right)}{3\left(D_{1}\left(D_{123}+D_{23}\right)+T d_{1} D_{23}\right)} .
\end{aligned}
$$

Substituting to (13) the parameter with actual value above, we obtain that $N V I_{1}=$ $0.94 \%$. Now it's fair to say that under the initial setting, value of information is quite small if we compare with the total amount. But if we say that the payoff is counted by billion, then $0.94 \%$ still makes a lot difference. Hence it has to be decided by the real situation.

The described method of calculation of NVI in the form (13) can be extended for the cooperative case of the game:

$$
N V I_{i}{ }^{C}=\frac{\xi_{i}-\xi_{i}^{*}}{\xi_{i}} \times 100 \%,
$$

where $\left\{\xi_{i}\right\}_{i=1}^{n}$ is the cooperative solution (Shapley value, $\tau$-value etc.) of the game computed under conditions of the complete information and $\left\{\xi_{i}^{*}\right\}_{i=1}^{n}$ is the cooperative solution calculated with a lack of information.

\section{Value of information for uncertainty of possible adjustment of upper boundary of control}

In classical differential game theory (Petrosjan, 1985), tasks are commonly considered without changing the structure. In (Gromova and Petrosian, 2016) differential game of pollution control embodies the dynamic update of information. (Gromov and Gromova, 2017) explores a hybrid differential game of pollution control with regime switching. While in our case, we are analyzing the model of industrial production, in which at some point the upper limit of the permissible level of 
contamination which caused by production is possible to change. Under this condition, we are studying the maintaining optimal controls and optimal trajectories. Based on that, value of information is obtained.

We simplify the model above and consider the process of pollution with only one player. We assume that pollution is proportional to the volume of production and the rate of emissions $u$ in the atmosphere is most likely restrained by the government or other parties. The strategy for player is to select optimal control for the purpose of maximal payoff. The model we consider can be generalized for the case of non-cooperative or cooperative differential game.

The dynamic is defined by the equation:

$$
\dot{x}=u, \quad u \in[0, b], \quad x(0)=x_{0} .
$$

The maximization of business owner's payoff is obtained:

$$
J\left(x_{0}, T, u\right)=\int_{0}^{T}\left(\left(b-\frac{1}{2} u\right) u-d x\right) d t \rightarrow \max _{u} .
$$

Suppose that at time moment $\tau_{s} \in[0, T]$, the upper boundary of control changes to $\bar{b}$, i.e. for $t \in\left[\tau_{s}, T\right]$ the control satisfies the constraint $u(t) \in[0, \bar{b}]$.

The Hamiltonian is:

$$
H(x, u, \psi)=\left(b-\frac{u}{2}\right) u-d x+\psi u,
$$

The optimal control:

$$
\begin{gathered}
\frac{\partial H}{\partial u_{i}}=b-u+\psi=0, \\
u^{*}=b+\psi .
\end{gathered}
$$

Because the second derivative of $H$ at $u=u_{i}^{*}$ is $-1<0$, so we confirm that we get the maximal Hamiltonian function at optimal control $u^{*}$.

The adjoint equations:

$$
\frac{\partial \psi}{\partial t}=-\frac{\partial H}{\partial x}=d
$$

And The endpoint $x(T)$ is free,

$$
\begin{gathered}
\psi(t)=d t+C, \\
\psi(T)=0=d T+C, \quad C=-d T, \\
\psi(t)=d(t-T) .
\end{gathered}
$$

The optimal control is:

$$
u^{*}=b+d(t-T) .
$$

Since the sign of control has to be non-negative and when $u^{*}=b+d(t-T)=0$, we have $t=T-\frac{b}{d}$. Then the optimal control $u^{*}$ should be reorganized as:

$$
u^{*}(t)= \begin{cases}0, & t \in\left[0, T-\frac{b}{d}\right] \\ b+d(t-T), & t \in\left[T-\frac{b}{d}, T\right] .\end{cases}
$$




\subsection{For the case with a change in upper limit}

Since the optimal control does not exceed $b$ for any $t \in[0, T]$, an increase in the upper limit of the emission level $b \rightarrow \bar{b}$ will not lead to the change the optimal control. However, if the updated upper boundary of control is less than $b$, i.e. $\bar{b}<b$, then the control will take the form:

$$
u^{*}(t)= \begin{cases}b+d(t-T), & t \in\left[T-\frac{b}{d}, \theta\right], \\ \bar{b}, & t \in[\theta, T]\end{cases}
$$

where $b+d(\theta-T)=\bar{b}, \theta=T+\frac{\bar{b}-b}{d} \geq \tau_{s}$ and obviously $u^{*}(t)=0, t \in\left[0, T-\frac{b}{d}\right]$. Besides, let $b+d\left(\tau_{s}-T\right)>0$, so the optimal control won't reach the lower boundary.

\subsection{The adjustment of upper boundary is unknown}

The business owner has no idea of the change of upper limit of control, then he will suffer the loss in size $P \int_{\theta}^{T}(b+d(t-T)-\bar{b}) d t$ in which $P$ is a penalty coefficient. Then the new payoff is formulated:

$$
J\left(x_{0}, T, u\right)=\int_{0}^{T}\left(\left(b-\frac{1}{2} u\right) u-d x\right) d t-P \int_{\theta}^{T}(b+d(t-T)-\bar{b}) d t \rightarrow \max ,
$$

Thing has to be clear that the owner will not take the loss unless he reaches the point where the condition is changed. Since the owner is not aware of change, we assume he will follow the routine. Therefore the optimal control will be identical to the previous:

$$
u_{1}^{*}(t)=\left\{\begin{array}{lr}
0, & t \in\left[0, T-\frac{b}{d}\right] . \\
b+d(t-T), & t \in\left[T-\frac{b}{d}, T\right] .
\end{array}\right.
$$

The expression for the optimal trajectory can be acquired by calculating the integral of $\dot{x}$ as shown in (15),

$$
x_{1}^{*}(t)= \begin{cases}x_{0}, & t \in\left[0, T-\frac{b}{d}\right] . \\ (b-d T) t+\frac{d}{2} t^{2}+C_{1}, & t \in\left[T-\frac{b}{d}, T\right],\end{cases}
$$

where $C_{1}=x_{0}-b T+\frac{b^{2}}{2 d}+\frac{d T^{2}}{2}$.

Then the total payoff:

$$
\begin{aligned}
J_{1}^{*} & =\int_{0}^{T-\frac{b}{d}}\left((b-0) 0-d x_{0}\right) d t-P \int_{\theta}^{T}((b+d(t-T)-\bar{b}) d t \\
& +\int_{T-\frac{b}{d}}^{T}\left(\left(b-\frac{1}{2}(b+d(t-T))\right)(b+d(t-T))-d\left((b-d T) t+\frac{d}{2} t^{2}+C_{1}\right)\right) d t \\
& =-d x_{0}\left(T-\frac{b}{d}\right)-\frac{d^{2}}{3}\left(T^{3}-\left(T-\frac{b}{d}\right)^{3}\right)+\left(d^{2} T-\frac{b d}{2}\right)\left(T^{2}-\left(T-\frac{b}{d}\right)^{2}\right)+ \\
& +\left(\frac{b^{2}}{2}-\frac{d^{2} T^{2}}{2}-d C_{1}\right) \frac{b}{d}-P \frac{(b-\bar{b})^{2}}{2 d} .
\end{aligned}
$$

3.3. The adjustment of upper boundary is known $b \rightarrow \bar{b}$.

To calculate the optimal control, it is necessary to consider optimal control problem separately on two intervals, starting from the second. On the interval $\left[\tau_{s}, T\right]$, we define the optimal value function $J_{2^{\prime}}$

$$
J_{2^{\prime}}(x, u)=\int_{\tau_{s}}^{T}\left(\left(b-\frac{1}{2} u\right) u-d x\right) d t
$$


where $u(t)$ is defined like previous $u_{1}^{*}(t)$ under several intervals and $x(t)$ satisfies the condition in (15) with $x\left(\tau_{s}\right)=x_{s}$.

We obtain the value of the owner's payoff on the second interval $J_{2^{\prime}}^{*}$, which for a fixed $\tau_{s}$ will depend only on $x_{s}$. Now we consider the first interval $\left[0, \tau_{s}\right]$. At the moment of time $\tau_{s}$, the end of the trajectory will not be fixed, but since it is necessary to take into account the game in the second interval. We will consider the total payoff in mixed form (integral + terminal at the time of change $\tau_{s}$ ):

$$
J\left(x_{0}, T, u\right)=\int_{0}^{\tau_{s}}\left(\left(b-\frac{1}{2} u\right) u-d x\right) d t+J_{2^{\prime}}^{*}\left(x_{s}\right) \rightarrow \max .
$$

In this case for $J_{2}^{*}$ :

$$
\begin{aligned}
J_{2^{\prime}}^{*} & =\int_{\tau_{s}}^{T+\frac{\bar{b}-b}{d}}\left(\left(b-\frac{1}{2}(b+d(t-T))\right)(b+d(t-T))-d x\right) d t+ \\
& +\int_{T+\frac{\bar{b}-b}{d}}^{T}\left(\left(b-\frac{1}{2} \bar{b}\right) \bar{b}-d x\right) d t \\
& =\int_{\tau_{s}}^{T+\frac{\bar{b}-b}{d}}(-d x) d t+\int_{T+\frac{\bar{b}-b}{d}}^{T}(-d x) d t=-d x\left(T-\tau_{s}\right) .
\end{aligned}
$$

In order to write down the conjugate equations and conditions of transversality, we calculate a partial derivative of terminal winnings $J_{2^{\prime}}^{*}\left(x_{s}\right)$ on $x_{s}$ :

$$
\frac{\partial J_{2^{\prime}}^{*}}{\partial x_{s}}=-d\left(T-\tau_{s}\right)
$$

In this case, we get the value of $\psi$ at the end of the first interval $\tau_{s}: \psi\left(\tau_{s}\right)=$ $-d\left(T-\tau_{s}\right)$.

In this case, the composite control equation will be written as follows:

$$
u_{2}^{*}(t)= \begin{cases}0, & t \in\left[0, \theta-\frac{\bar{b}}{d}\right] . \\ b+d(t-T), & t \in\left[\theta-\frac{\bar{b}}{d}, \theta\right], \\ \bar{b}, & t \in[\theta, T],\end{cases}
$$

where $\theta=T+\frac{\bar{b}-b}{d}$.

Correspondingly, the optimal trajectory:

$$
x_{2}^{*}(t)=\left\{\begin{array}{lrl}
x_{0}, & t \in\left[0, \theta-\frac{\bar{b}}{d}\right] . \\
(b-d T) t+\frac{d}{2} t^{2}+C_{1}, & t \in\left[\theta-\frac{\bar{b}}{d}, \theta\right], \\
\bar{b} t+C_{2}, & t \in[\theta, T] .
\end{array}\right.
$$

where $C_{1}=x_{0}-b T+\frac{b^{2}}{2 d}+\frac{d T^{2}}{2}$ and $C_{2}=C_{1}+(b-d T) \theta+\frac{d}{2} \theta^{2}-\bar{b} \theta$.

Then under this condition, the total payoff:

$$
\begin{aligned}
J_{2}^{*} & =\int_{0}^{T-\frac{b}{d}}\left((b-0) 0-d x_{0}\right) d t+\int_{\theta}^{T}\left(\left(b-\frac{1}{2} \bar{b}\right) \bar{b}-d\left(\bar{b} t+C_{2}\right)\right) d t \\
& +\int_{T-\frac{b}{d}}^{\theta}\left(\left(b-\frac{1}{2}(b+d(t-T))\right)(b+d(t-T))-d\left((b-d T) t+\frac{d}{2} t^{2}+C_{1}\right)\right) d t \\
& =-d x_{0}\left(T-\frac{b}{d}\right)-\frac{d^{2}}{3}\left(\theta^{3}-\left(T-\frac{b}{d}\right)^{3}\right)+\left(d^{2} T-\frac{b d}{2}\right)\left(\theta^{2}-\left(T-\frac{b}{d}\right)^{2}\right)+ \\
& +\left(\frac{b^{2}}{2}-\frac{d^{2} T^{2}}{2}-d C_{1}\right)\left(\theta-T+\frac{b}{d}\right)+\left(b \bar{b}-\frac{\bar{b}^{2}}{2}-d C_{2}\right)(T-\theta)-\frac{d \bar{b}}{2}\left(T^{2}-\theta^{2}\right) .
\end{aligned}
$$


Suppose $T=30, b=20, d=1, x_{0}=10, \bar{b}=10, P=10, \tau_{s}=15$ for both cases. As shown in the figures below, we have to admit that the uncertainty of upper boundary indeed leads to the various performance of their optimal controls and trajectories. And it's quite obvious that being aware of change of upper limit is better. However, we are not able to quantify the actual worth of value of information currently. We will discuss it in the following subsection.

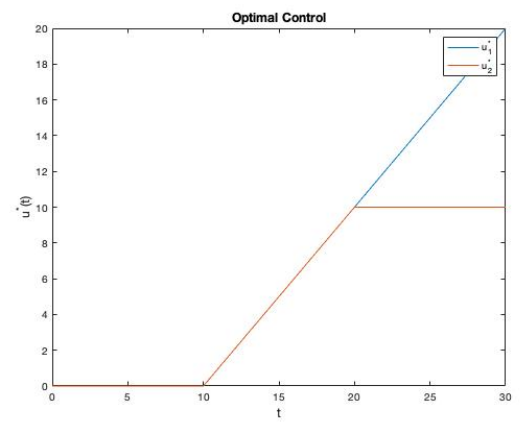

Fig. 3. Optimal control for two cases

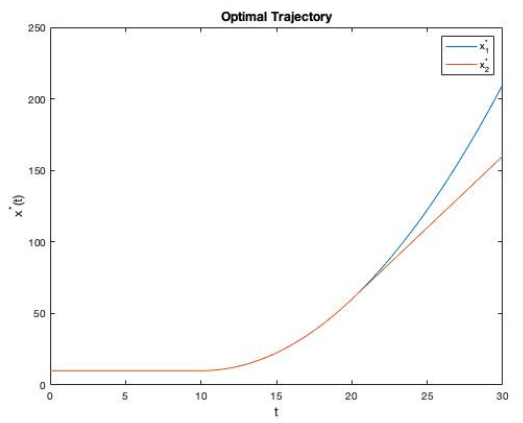

Fig. 4. Optimal trajectory for two cases

\subsection{Evaluation of value of information - VI}

For evaluating the characteristic value of information, we propose the new characteristic which is denoted as NVI:

$$
\begin{aligned}
& N V I=\frac{J_{2}^{*}-J_{1}^{*}}{J_{2}^{*}} \times 100 \% \\
& =\left(\frac{d^{2}\left(\left(T-\frac{b}{d}\right)^{3}-\left(T+\frac{\bar{b}-b}{d}\right)^{3}\right)}{3}-\frac{d^{2}\left(\left(T-\frac{b}{d}\right)^{3}-T^{3}\right)}{3}+\right. \\
& +\left(\left(T-\frac{b}{d}\right)^{2}-\left(T+\frac{\bar{b}-b}{d}\right)^{2}\right)\left(\frac{b d}{2}-T d^{2}\right)-\left(\left(T-\frac{b}{d}\right)^{2}-T^{2}\right)\left(\frac{b d}{2}-T d^{2}\right)- \\
& -\frac{\bar{b}}{d}\left(d\left(\frac{d T^{2}}{2}-T b+\frac{d b^{2}}{2}+x_{0}\right)-\frac{b^{2}}{2}+\frac{T^{2} d^{2}}{2}\right)+\frac{\bar{b} d\left(\left(T+\frac{\bar{b}-b}{d}\right)^{2}-T^{2}\right)}{2}+ \\
& +\frac{P(\bar{b}-b)^{2}}{2 d}+\frac{\bar{b}-b}{d}\left(d \left(x_{0}-T b+d \frac{\left(T+\frac{\bar{b}-b}{d}\right)^{2}}{2}+\left(T+\frac{\bar{b}-b}{d}\right)(b-T d)+\right.\right. \\
& \left.\left.+\frac{T^{2} d}{2}+\frac{b^{2} d}{2}-\bar{b}\left(T+\frac{\bar{b}-b}{d}\right)\right)-\bar{b} b+\frac{\bar{b}^{2}}{2}\right)+\frac{b}{d}\left(d\left(\frac{d T^{2}}{2}-T b+\frac{d b^{2}}{2}+x_{0}\right)-\frac{d^{2}}{2}\right)+ \\
& \left.\left.+\frac{T^{2} d^{2}}{2}\right)\right) /\left(\frac{d^{2}\left(\left(T-\frac{b}{d}\right)^{3}-\left(T+\frac{\bar{b}-b}{d}\right)^{3}\right)}{3}+\left(\left(T-\frac{b}{d}\right)^{2}-\left(T+\frac{\bar{b}-b}{d}\right)^{2}\right)\left(\frac{b d}{2}-\frac{T d}{2}\right)\right) \\
& -\frac{\bar{b}}{d}\left(d\left(\frac{d T^{2}}{2}-T b+\frac{b d^{2}}{2} x_{0}\right)-\frac{b^{2}}{2}+\frac{T^{2} d^{2}}{2}\right)+\frac{\bar{b} d}{2}\left(\left(T+\frac{\bar{b}-b}{d}\right)^{2}-T^{2}\right)+ \\
& +\frac{\bar{b}-b}{b}\left(d \left(x_{0}-T b+\frac{d}{2}\left(T+\frac{\bar{b}-b}{2}\right)^{2}+\left(T+\frac{\bar{b}-b}{d}\right)(b-T d)+\frac{T^{2} d}{2}+\frac{b^{2} d}{2}-\right.\right. \\
& \left.\left.-\bar{b}\left(T+\frac{\bar{b}-b}{d}\right)\right)-\bar{b} b+\frac{\bar{b}^{2}}{2}-d x_{0}\left(T-\frac{b}{d}\right)\right) \times 100 \% \text {. }
\end{aligned}
$$


Assume that the value of parameter is same to the previous setting, in this case, we get $N V I=38.46 \%$ which indicates that under current circumstance, value of information is pretty crucial. The unawareness of adjustment of upper limit may dramatically influence the business by reducing close to $40 \%$ of theoretical profit when the information is known.

\section{Conclusion}

In the paper two new models of the pollution emissions control with a lack of information was considered and the new characteristic such that the normalized value of information was proposed.

\section{References}

Basar, T. and Olsder, G. J. (1998). Dynamic noncooperative game theory. In: Society for Industrial and Applied Mathematics.

Bondarev, A. and Gromov, D. (2021). On the structure and regularity of optimal solutions in a differential game with regime switching and spillovers. In: Dynamic Economic Problems with Regime Switches. Dynamic Modeling and Econometrics in Economics and Finance. Springer. Vol. 25. pp. 187-208.

Dockner, E. J., Jorgensen, S., Van Long, N., \& Sorger, G. (2000). Differential games in economics and management science. Cambridge University Press.

Gamkrelidez, R. V. (1999). Discovery of the maximum principle. Journal of dynamical and control systems, 5(4), 437-451.

Gromov, D. and Gromova, E. (2017). On a class of hybrid differential games. Dynamic Games and Applications, 7(2), 266-288.

Gromova, E. (2016). The Shapley value as a sustainable cooperative solution in differential games of three players. In: Recent Advances in Game Theory and Applications, Static and Dynamic Game Theory: Foundations and Applications. pp. 67-91.

Gromova, E. and Petrosian, O. (2016). Control of information horizon for cooperative differential game of pollution control. In: International Conference - Stability and Oscillations of Nonlinear Control Systems (Pyatnitskiy's Conference). pp. 1-4, doi: 10.1109/STAB.2016.7541187.

Petrosjan, L. A. (1985). Cooperative differential games and their applications. Petrosjan L. A, Danilov. N. N. - Tmosk: Tomsk State University Publishing House, pp. 273.

Pontryagin, L.S. (1961). Mathematical theory of optimal processes. Moscow: Science, pp. 392.

Raiffa, H. and R. Schlaifer (1961). Applied statistical decision theory. New York, Wiley.

Tur, A., Gromova, V. (2018). On the optimal control of pollution emissions for the largest enterprises of the Irkutsk region of the Russian Federation. Mat. Teor. Igr Pril., 10:2 (2018), 62-89; Autom. Remote Control, 81:3 (2020), 548-565. 